\title{
Recognizing People's Faces: from Human to Machine Vision
}

\author{
Massimo Tistarelli \\ Computer Vision Laboratory \\ DAP - University of Sassari \\ piazza Duomo 6 - 07041 Alghero (SS) - Italy \\ Email: tista@uniss.it
}

\author{
Manuele Bicego and Enrico Grosso \\ Computer Vision Laboratory \\ DEIR - University of Sassari \\ via Torre Tonda 34 - 07100 Sassari - Italy \\ Email: bicego@uniss.it; grosso@uniss.it
}

\begin{abstract}
As confirmed by recent neurophysiological studies, the use of dynamic information is extremely important for humans in visual perception of biological forms and motion. Apart from the mere computation of the visual motion of the viewed objects, the motion itself conveys far more information, which helps understanding the scene. This paper provides an overview and some new insights on the use of dynamic visual information for face recognition. In this context, not only physical features emerge in the face representation, but also behavioral features should be accounted. While physical features are obtained from the subject's face appearance, behavioral features are obtained from the individual motion and articulation of the face. In order to capture both the face appearance and the face dynamics, a dynamical face model based on a combination of Hidden Markov Models is presented. The number of states (or facial expressions) are automatically determined from the data by unsupervised clustering of expressions of faces in the video. The underlying architecture closely recalls the neural patterns activated in the perception of moving faces. Preliminary results on real video image data show the feasibility of the proposed approach.
\end{abstract}

Keywords-Face recognition, Biometrics, Human visual system, Human perception

\section{INTRODUCTION}

Because of its natural interpretation (human visual recognition is mostly based on face analysis) and the low intrusiveness, face-based recognition, among others, is one of the most important biometric trait. There are many lessons we may learn from natural systems. Among them the use of minimal energy is a natural principle of paramount importance. Many natural mechanisms strongly rely on this principle. This paper highlights some basic principles related to living systems to gather insights on sensory data acquisition and processing for recognition [1].

Recently, the analysis of video streams of face images has received an increasing attention [2], [3], [4], [5], [6], [7], [8], [9]. A first advantage in using video is the possibility of employing redundancy present in the video sequence to improve still images recognition systems. One example is the use of voting schemes to combine results obtained for all the faces in the video, or the choice of the faces best suited for the recognition process. Another possibility is to use video se- quence to build a 3D representation or super-resolution images. Besides these motivations, recent psychophysical and neural studies [1], [10] have shown that dynamic information is very crucial in the human face recognition process. These findings inspired the development of true spatio-temporal video-based face recognition systems [2], [3], [4], [5], [6], [7], [8], [9].

The example system described in this paper makes use of physiological and behavioral visual cues for person authentication, based on pseudo hierarchical Hidden Markov Models (HMM). The method is based on the modeling of the entire video sequence with an HMM in which the emission probability function of each state consists in another HMM itself (see Fig. 5), resulting in a pseudo-hierarchical HMM. This complex structure represents a well founded, fully probabilistic, approach to face perception based on video modeling.

Several comparative examples are presented showing the advantages of processing animated face video sequences.

\section{NEUROPHYSIOLOGY AND INFORMATION PROCESSING}

Neural systems that mediate face recognition appear to exist very early in life. In normal infancy, the face holds particular significance and provides nonverbal information important for communication and survival [11].

Face recognition ability is present during the first 6 months of life, while a visual preference for faces and the capacity for very rapid face recognition are present at birth [12], [13]. By 4 months, infants recognize upright faces better than upside down faces, and at 6 months, infants show differential eventrelated brain potentials to familiar versus unfamiliar faces [14], [15]. Apart from speech, face analysis is certainly the first and major biometric cue used by humans and therefore very important to study accurately.

Early studies on face recognition in primates revealed a consistent neural activity in well identified areas of the brain, mainly involving the temporal sensory area. More recent research revealed that this is not the case, but many different brain areas are taken into play at different stages of face analysis and recognition. This also recalls the need for a very complex representation including both photometric and dynamic information on the facial characteristics. 


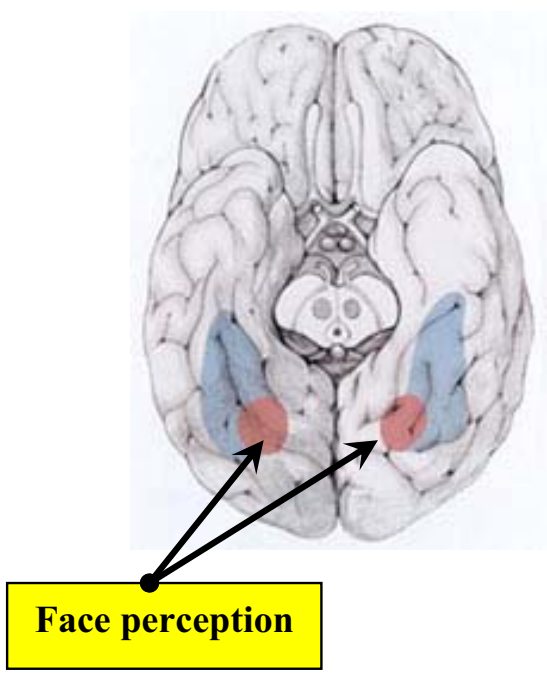

Fig. 1. Schema of the human brain as seen from below. The highlighted areas are those initially devoted to the perception of faces and object's form.

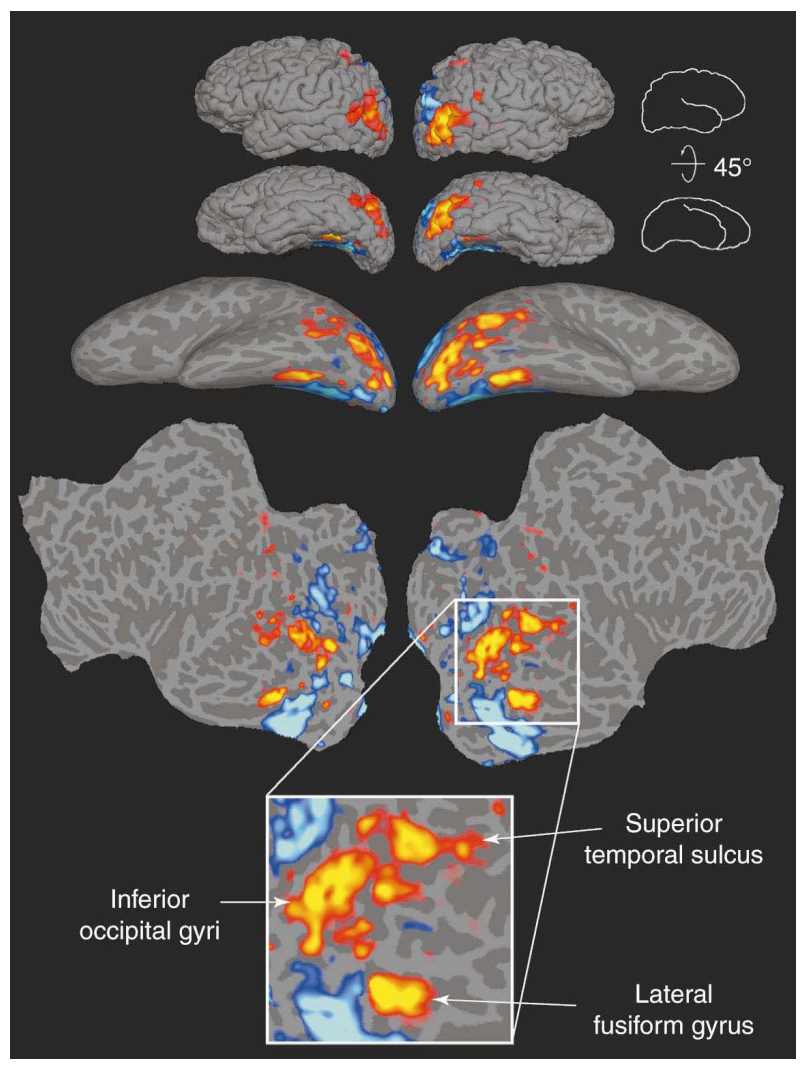

Fig. 2. Activation areas from fMRI responding mainly to face stimuli (red to yellow patterns) or to house pictures (blue patterns). (Reproduced from [24])

\section{A. Neural mapping of face representations}

Much is known about the neural systems that subserve face recognition in adult humans and primates. Face-selective neurons have been found in the inferior temporal areas (TEa and TEm), the superior temporal sensory area, the amygdala, the ventral striatum (which receives input from the amygdala) and the inferior convexity [16]. Using functional magnetic resonance imaging (fMRI), an area in the fusiform gyrus was found significantly activated when the subjects viewed faces [17], [18], [19]. Within this "general face activation area" specific regions of interest have been reported responding significantly more strongly to passive viewing of facespecific stimuli. An fMRI study on individuals with autism and Asperger syndrome a failure to activate the fusiform face area during face processing. Damage to fusiform gyrus and to amygdala results in impaired face recognition [20], [21]. Parts of the inferior and medial temporal cortex may work together to process faces. For example, the anterior inferior temporal cortex and the superior temporal sulcus project to the lateral nucleus of the amygdala, with the amygdala responsible for assigning affective significance to faces, and thus affecting both attention and mnemonic aspects of face processing [22], [23].

A recent fMRI analysis on the neural architecture subduing face perception, revealed an interesting relation between perceptual task and neural activation. It seems that face-sensitive areas are involved also in the recognition of non-face objects such as houses, cars and animals, while specific tasks related to faces also involve non-face areas in the brain [24]. This study suggests a double architecture for face perception, formed by two connected neural activation patterns: the former devoted to process static, unchanging and invariant features of the face; the latter devoted to the analysis of changing features in the face. This architecture is also in agreement with other works on the recognition of biological motion and its relation to face perception [1], [25]. Knight et al. discovered that motion improves face recognition in difficult tasks (i.e. when 3D features are missing or the face pose is unexpected). The work by Vaina et al. also relates motion perception to face-related visual tasks. The presented fMRI study reveals a multiple activation whenever the perceptual task involves motion and shape recognition of living creatures. This also implies that the neural activation is not limited to a fixed pattern, but more strongly depends on the visual task than on the viewed subject.

\section{B. Relevance of the time dimension}

The high specialization of specific brain areas for face analysis and recognition motivates the relevance of faces for social relations. On the other hand, this suggests that face understanding is not a low level process but involves higher level functional areas in the brain. These, in turn, must rely on a rich series of low level processes applied to enhance and extract face-specific features. Facial features are not simply distinctive points on the segmented face, but rather a collection of image features representing specific (and anatomically stable) areas of the face such as the eyes, eyebrows, ears, mouth, nostrils etc. Other, subject-specific, features are also included, such as the most famous Marilyn Monroe's naevus [26], [27].

As shown by Vaina et al. [25], the visual task strongly influences the areas activated during visual processing. This is specially true for face perception, where not only face-specific 


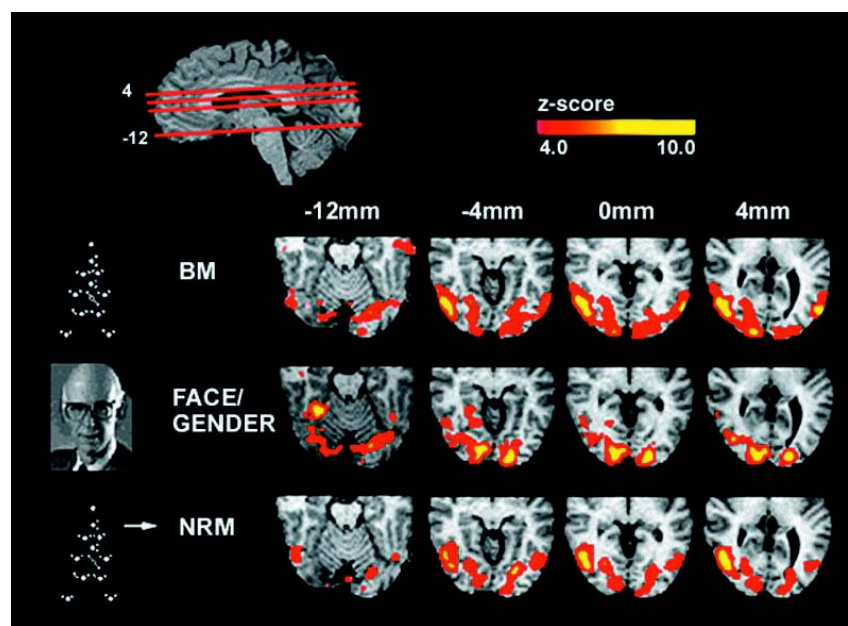

Fig. 3. Activation areas from fMRI responding to: biological motion (top), gender estimation from face (middle), non-rigid motion (bottom). The red lines on the top picture indicate the position of the 4 axial slices spanning between $-12 \mathrm{~mm}$ and $4 \mathrm{~mm}$, with respect to the central position. On the left a schematic representation of the presented stimuli is shown: moving light dots are used for motion stimuli and face pictures for face stimuli (Reproduced from [25]).

areas are involved, but a consistent neural activity is registered in brain areas devoted to motion perception and gaze control.

The time dimension is involved also when unexpected stimuli are presented [1]. Humans can easily recognize faces which are rotated and distorted up to a limited extent. The increase in time reported for recognition of rotated and distorted faces implies: the expectation on the geometric arrangement of facial features, and a specific process to organize the features (analogous to image registration and warping) before the actual recognition process can take place. On the other hand, it has been shown that the recognition error for an upside-down face decreases when the face is shown in motion [1].

From the basic element related to the face shape and color, subduing a multi-area neural activity, cognitive processes are started not only to determine the subject's identity, but also to understand more abstract elements (even uncorrelated to the subject's identity) which characterize the observed person (age, race, gender, emotion etc.). These, in turn, also recall taskspecific processes, such as motion analysis and facial features tracking for understanding emotion-specific patterns [25], [28], [29], [30], [31], [32], [33].

\section{VIDEO-BASED FACE IMAGE ANALYSIS}

Conversely to previous assumptions and theories of human neural activity, face perception does not involve a single, well defined area of the brain. It seems that the traditional "face area" is responsible for the general shape analysis but it is not sufficient for recognition as well for other tasks. In the same way, face recognition by computers can not be seen as a single, monolithic process, but several representations must be devised into a multi-layered architecture.

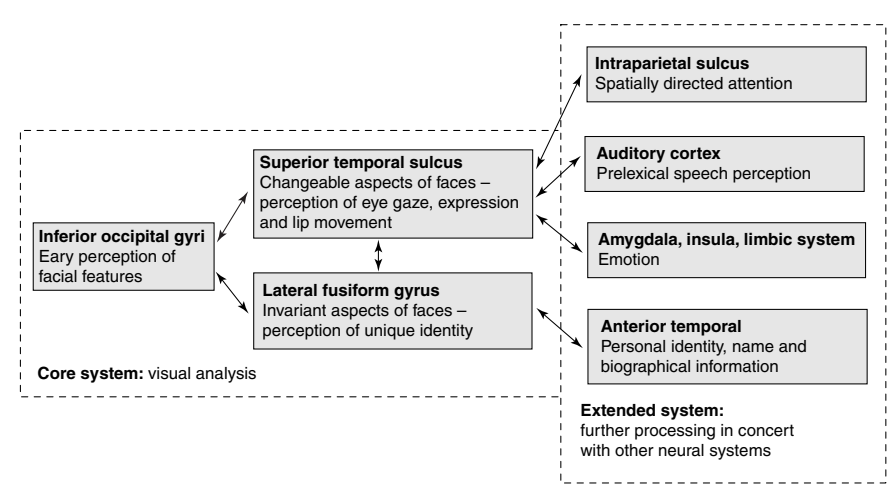

Fig. 4. A model of the distributed neural system for face perception (reproduced from [24]).

An interesting approach to multi-layer face processing has been proposed by Haxby [24]. The proposed architecture (sketched in figure 4) divides the face perception process into two main layers: the former devoted to the extraction of basic facial features and the latter processing more changeable facial features such as lip movements and expressions.

It is worth noting that the encoding of changeable features of the face also captures some behavioral features of the subject, i.e. how the facial traits are changed according to a specific task or emotion.

This double layered architecture can be represented by two distinct but similar processing units devoted to two distinct tasks. The system proposed in the remainder of the paper proposes the use of the Hidden Markov Models as elementary units to build a double layer architecture to extract shape and motion information from face sequences. The architecture is based on a multi-dimensional HMM which is capable of both capturing the shape information and the change in appearance of the face. This multi-layer architecture was termed Pseudo Hierarchical Hidden Markov Model to emphasize the hierarchical nature of the process involved [34].

\section{Hidden Markov Models and Pseudo Hierarchical HidDEN MARKOV MOdElS}

A discrete-time Hidden Markov Model $\boldsymbol{\lambda}$ can be viewed as a Markov model whose states cannot be explicitly observed. A probability distribution function is associated to each state, modeling the probability of emitting symbols from that state [35]:

- $H=\left\{H_{1}, H_{2}, \cdots, H_{K}\right\}$ the finite set of the possible hidden states;

- the transition matrix $\mathbf{A}=\left\{a_{i j}, 1 \leq j \leq K\right\}$ representing the probability to go from state $H_{i}$ to state $H_{j}$;

- the emission matrix $\mathbf{B}=\left\{b\left(o \mid H_{j}\right)\right\}$, indicating the probability of the emission of the symbol $o$ when system state is $H_{j}$; typically continuous HMM were employed: $b\left(o \mid H_{j}\right)$ is represented by a Gaussian distribution;

- $\boldsymbol{\pi}=\left\{\pi_{i}\right\}$, the initial state probability distribution, representing probabilities of initial states; 
For convenience, we denote an HMM as a triplet $\boldsymbol{\lambda}=$ $(\mathbf{A}, \mathbf{B}, \boldsymbol{\pi})$.

Given a set of sequences $\left\{S^{k}\right\}$, the training of the model is usually performed using the standard Baum-Welch reestimation [35]. During the training phase, the parameters $(\mathbf{A}, \mathbf{B}, \boldsymbol{\pi})$ that maximize the probability $P\left(\left\{S^{k}\right\} \mid \boldsymbol{\lambda}\right)$ are computed. The evaluation step (i.e. the computation of the probability $P(S \mid \boldsymbol{\lambda})$, given a model $\boldsymbol{\lambda}$ and a sequence $S$ to be evaluated) is performed using the forward-backward procedure [35].

\section{A. Pseudo Hierarchical-HMM}

The emission probability of a standard HMM is typically modelled using simple probability distributions, like Gaussians or Mixture of Gaussians. Nevertheless, in the case of sequences of face images, each symbol of the sequence is a face image, and a simple Gaussian may not be sufficiently accurate to properly model the probability of emission. In the PH-HMM, the emission probability is modelled using another HMM, which has been proven to be very accurate in describing faces [36], [37], [38], [39].

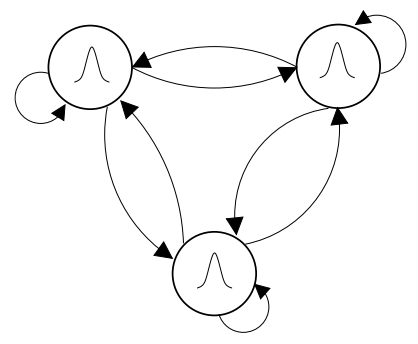

(a)

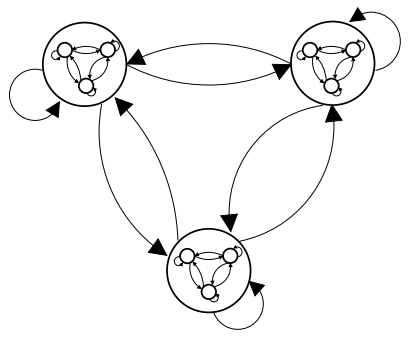

(b)
Fig. 5. Differences between standard HMMs and PH-HMM, where emission probabilities are displayed into the state. (a) Standard Gaussian emission. (b) Pseudo Hierarchical HMM, where the emissions are HMMs.

Fixed the number of states $K$ of the PH-HMM, for each class $C$ the training is performed in two sequential steps:

1) Training of emission. The first level sequence $S^{k}=$ $s_{1}^{k}, s_{2}^{k}, \cdots, s_{T}^{k}$ is "unrolled", i.e. the $\left\{s_{i}^{k}\right\}$ are considered to form an unordered set $U$ (no matter the order in which they appear in the first level sequence). This set is subsequently split in $K$ clusters, grouping together similar $\left\{s_{i}^{k}\right\}$. For each cluster $j$, a standard HMM $\boldsymbol{\lambda}_{j}$ is trained, using the second-level sequences contained in that cluster. These HMMs $\lambda_{j}$ represents the emission HMMs.

2) Training of transition and initial states matrices. Considering that the emission probability functions are determined by the emission HMMs, the transition and the initial states probability matrices of the PH-HMM are estimated using the first level sequences. The number of clusters determines the number of the PH-HMM states. In this phase, only the transition matrix and the initial state probability are estimated, since the emission has been already determined in the previous step.
Because of the sequential estimation of the PH-HMM components (firstly emission and then transition and initial state probabilities), the resulting HMM is a "pseudo" hierarchical HMM. In a truly hierarchical model, the parameters $\mathbf{A}, \boldsymbol{\pi}$ and B should be jointly estimated, because they could influence each other [41].

This complex mathematical and statistical model of the sequence of face images can be divided into two layers:

- the former, a straightforward HMM encoding of the face, is devoted to capture the face shape and its static, invariant features;

- the latter, obtained from a clustering process of standard HMMs, is applied to represent the temporal evolution of the face, thus modeling the changeable features of the face.

This dual layer model closely resembles the neural architecture hypothesized by Haxby et al. [24] and reproduce in figure 4

\section{Authentication of FACE SEQUENCES}

A biometric authentication system is based on two steps: enrollment and identity verification. Given few video sequences captured from the subject's face, the enrollment phase aims at determining the best PH-HMM modeling the subject's face appearance. This model encompasses both the invariant aspects of the face and its changeable features. Identity verification is performed by projecting a captured face video sequence on the PH-HMM model belonging to the claimed identity.

The enrollment process consists on a series of sequential steps (for simplicity we assume only one video sequence $S=$ $s_{1}, s_{2}, \cdots, s_{T}$, the generalization to more than one sequence is straightforward):

1) The video sequence $S$ is analyzed to detect all faces sharing similar expression, i.e. to find clusters of expressions. Firstly, each face image $s_{i}$ of the video sequence is reduced to a raster scan sequence of pixels, used to train a standard spatial HMM [36], [39]. The resulting face HMM models are clustered in different groups based on their similarities [42], [43]. Faces in the sequence with similar expression are grouped together, independently from their appearance in time. The number of different expressions are automatically determined from the data using the Bayesian Inference Criterion [44].

2) For each expression cluster, a spatial face HMM is trained. In this phase all the sequences of the cluster are used to train the HMM. At the end of the process, $K$ HMMs are trained. Each spatial HMM models a particular expression of the face in the video sequence. These models represents the emission probabilities functions of the PH-HMM.

3) The transition matrix and the initial state probability of the PH-HMM are estimated from the sequence $S=$ $s_{1}, s_{2}, \cdots, s_{T}$, using the Baum-Welch procedure and the emission probabilities found in the previous step (see Sect. IV). This process aims at determining the temporal evolution of facial expressions over time. The number of 
states is fixed to the number of discovered clusters, this representing a sort of model selection criterion.

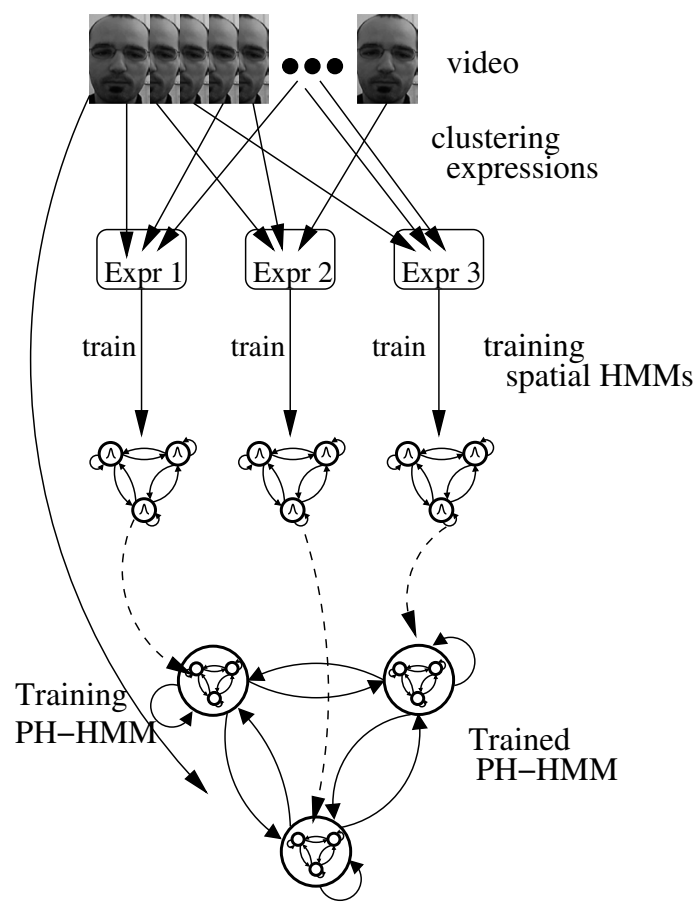

Fig. 6. Sketch of the enrollment phase of the proposed approach.

\section{A. Spatial HMM modeling: Analysis of face form}

The process to build spatial HMMs is used in two stages of the proposed algorithm: in clustering expressions, where one HMM is trained for each face, and in the PH-HMM emission probabilities estimation, where one HMM is trained for each cluster of faces. In the proposed approach, the local image structure is captured computing first and higher order statistics: the gray level mean, variance, Kurtosis and skewness (which are the third and the fourth moment of the data) [40].

After the image scanning and feature extraction process, a sequence of $D \times R$ features is obtained, where $D$ is the number of features extracted from each sub image (4), and $R$ is the number of image patches. The learning phase is then performed using standard Baum-Welch re-estimation algorithm [35]. In this case the emission probabilities are all Gaussians, and the number of states is set to be equal to four. The learning procedure is initialized using a Gaussian clustering process, and stopped after likelihood convergence.

\section{B. Clustering facial expressions}

The goal of this step is to group together all face images in the video sequence with the same appearance, namely the same facial expression, independently of its position within the sequence. It is worth noting that this process does not imply a segmentation of the sequence into homogeneous, contiguous fragments. The resulting sequences rather represent the temporal evolution of the changeable features in the subject's face.
Since each face is described with an HMM sequence, the expression clustering process is casted into clustering sequences represented by HMMs [45], [42], [46], [43]. Considering the unrolled set of faces $s_{1}, s_{2}, \cdots, s_{T}$, where each face $s_{i}$ is a sequence $s_{i}=o_{i 1}, o_{i 2}, \cdots, o_{i T_{i}}$, the clustering algorithm is based on the following steps:

1) Train one standard HMM $\boldsymbol{\lambda}_{i}$ for each sequence $s_{i}$.

2) Compute the distance matrix $D=\left\{D\left(s_{i}, s_{j}\right)\right\}$, where $D\left(s_{i}, s_{j}\right)$ is defined as:

$$
D\left(s_{i}, s_{j}\right)=\frac{P\left(s_{j} \mid \boldsymbol{\lambda}_{i}\right)+P\left(s_{i} \mid \boldsymbol{\lambda}_{j}\right)}{2}
$$

Since $\boldsymbol{\lambda}_{i}$ is trained using the sequence $s_{i}$, the closer is $s_{j}$ to $s_{i}$, the higher is the probability $P\left(s_{j} \mid \boldsymbol{\lambda}_{i}\right)$. This is not a quantitative but rather a qualitative measure of similarity [43], [42].

3) Given the similarity matrix $D$, a pairwise distancematrix-based method (e.g. an agglomerative method) is applied to perform the clustering [47].

It is impossible to arbitrarily establish the number of facial expressions in a sequence of facial images. Therefore, the number of clusters has been estimated from the data, using the standard Bayesian Inference Criterion (BIC) [44]. This is a penalized likelihood criterion which is able to find the best number of clusters as the compromise between the model fitting (HMM likelihood) and the model complexity (number of parameters).

\section{PH-HMM modeling: Analysis of temporal evolution}

From the extracted set of facial expressions, the $\mathrm{PH}$ HMM is trained. The different PH-HMM emission probability functions (spatial HMMs) model the facial expressions, while the temporal evolution of the facial expressions in the video sequence is modelled by the PH-HMM transition matrix. In particular, for each facial expression cluster, one spatial HMM is trained, using all faces belonging to the cluster (see section $\mathrm{V}-\mathrm{A}$ ). The transition and the initial state matrices are estimated using the procedure described in section IV. The number of states of the PH-HMM directly derives from the previous stage (number of clusters), representing a direct smart approach to the model selection issue.

\section{Face verification}

The verification of a subject's identity is straightforward. Captured a sequence of face images from an unknown subject, and a claimed identity, the sequence is fed to the corresponding PH-HMM, which returns a probability value. The claimed identity is verified if the computed probability value is over a predetermined threshold. This comparison corresponds to verifying if the captured face sequence is well modeled by the given PH-HMM.

\section{EXPERIMENTAL Results}

The system has been tested using a database composed of 21 subjects. Each subject is requested to vocalize ten digits, from one to ten. A minimum of five sequences for each subject have been acquired, in two different sessions. 


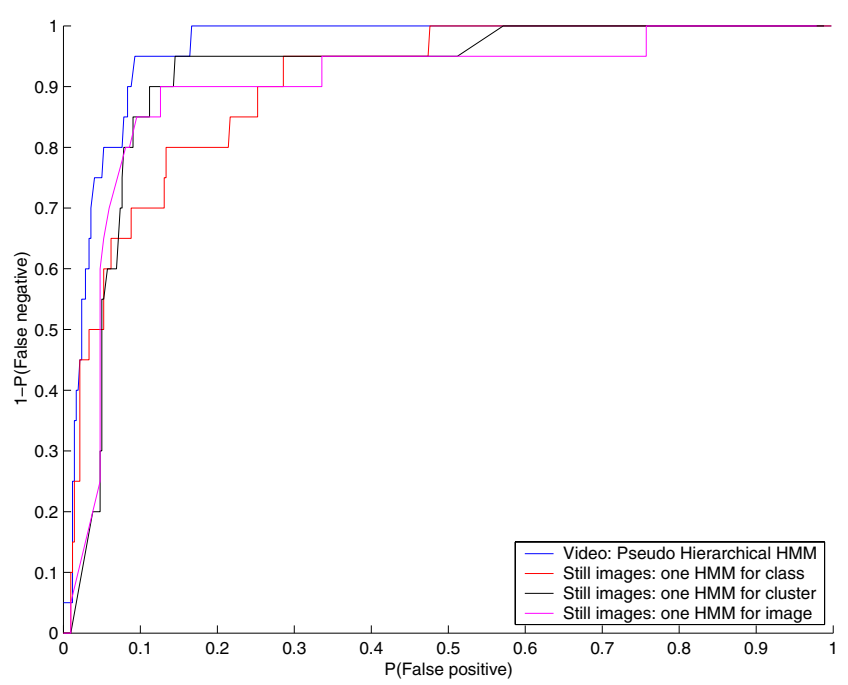

Fig. 7. The computed ROC curve for the verification experiment from video sequences of faces for the 4 methods reported

The proposed approach has been tested against three other HMM-based methods, which do not fully exploit the spatiotemporal information. The first method, called "1 HMM for all", applies one spatial HMM (as described in section VA) to model all images in the video sequence. The sum of the likelihoods represents the matching score. In the second method, called "1 HMM for cluster", one spatial HMM is trained for each expression cluster, using all the sequences belonging to that cluster. Given an unknown face video sequence, all images are fed into the different HMMs (and summed as before): the final matching score is the maximum among the different HMMs' scores. The last method, called "1 HMM for image", is based on training one HMM for each image in the video sequence. As in the "1 HMM for cluster" method, the matching score is computed as the maximum between the different HMMs' scores.

In all experiments only one video sequence for each subject has been used for the enrollment phase. Full client and impostor tests have been performed computing a ROC (Receiver Operating Curve). Testing and training sets were always disjoint, allowing a more reliable estimation of the error rate. In table I the Equal Error Rates (error when false positive and false negatives are equal) for the four methods are reported.

\begin{tabular}{|l|c|}
\hline Method & EER \\
\hline Still Image: 1 HMM for all & $20.24 \%$ \\
Still Image: 1 HMM for cluster & $10.60 \%$ \\
Still Image: 1 HMM for image & $13.81 \%$ \\
Video: PH-HMM & $9.40 \%$ \\
\hline
\end{tabular}

TABLE I

VERIFICATION RESULTS FOR THE REPORTED HMM-BASED, FACE MODELING METHODS.

From the comparative results reported in table 1 and 2

\begin{tabular}{|l|c|}
\hline Method & Accuracy \\
\hline Still Image: 1 HMM for all & $52.38 \%(11 / 21)$ \\
Still Image: 1 HMM for cluster & $66.67 \%(14 / 21)$ \\
Still Image: 1 HMM for image & $57.14 \%(12 / 21)$ \\
Video: PH-HMM & $90.48 \%(19 / 21)$ \\
\hline
\end{tabular}

TABLE II

IDENTIFICATION RESULTS (ACCURACY) FOR THE REPORTED HMM-BASED, FACE MODELING METHODS.

it can be noted that, including temporal information into the face model, a remarkable advantage is obtained. This finding confirms the importance of a dual architecture for face perception, where both static, invariant facial features and dynamic information are coupled to model the subject's appearance.

\section{CONCLUSIONS}

Despite of the simple neural architectures for face perception hypothesized in early neurological studies, the perception of human faces is a very complex task which involves several areas of the brain. The face perception process seems to involve several areas depending on the specific task required rather than on the nature of the stimulus. This task-driven model may be represented by a dual layer architecture where static and dynamic features are analysed separately to devise a unique face model.

The dual nature of the neural architecture allows to capture both static and dynamic data. As a consequence, not only physiological face features are processed, but also behavioral features, which are more related to how the face traits are changing over time. This last property is characteristic of each individual and implicitly represents the changeable features of the face.

A statistical model of the face appearance, which reflects the described dual-layered neural architecture, has been presented. In order to capture both static and dynamic features, the model is based on the analysis of face video sequences using Pseudo Hierarchical HMM. As for the PH-HMM model, the emission probability of each state is represented by another HMM. The number of states has been determined from the data by unsupervised clustering of facial expressions in the video. The resulting architecture is then capable of modeling both physiological and behavioral features, represented in the face image sequence and well represents the dual neural architecture described in [24].

Even though the experiments performed are very preliminary, already demonstrate the potential of the algorithm in coupling photometric appearance of the face and the temporal evolution of facial expressions. The proposed approach can be very effective in face identification or verification to exploit the subject's cooperation in order to enforce the required behavioral features and strengthen the discrimination power of a biometric system. 


\section{ACKNOWLEDGMENT}

This work was supported by funds from the European Union (European Network of Excellence Biosecure) and from funds of the Italian Ministry of Research.

\section{REFERENCES}

[1] B. Knight and A. Johnston. The role of movement in face recognition. Visual Cognition, 4:265-274, 1997.

[2] O. Yamaguchi, K. Fukui, and K. Maeda. Face recognition using temporal image sequence. In Proc. Int. Conf. on Automatic Face and Gesture Recognition, 1998.

[3] Z. Biuk and S. Loncaric. Face recognition from multi-pose image sequence. In Proc. of Int. Symp. on Image and Signal Processing and Analysis, 2001.

[4] Y. Li. Dynamic face models: construction and applications. PhD thesis, Queen Mary, University of London, 2001.

[5] G. Shakhnarovich, J. W. Fisher, and T. Darrell. Face recognition from long-term observations. In Proc. of European Conf. on Computer Vision, 2002.

[6] S. Zhou, V. Krueger, and R. Chellappa. Probabilistic recognition of human faces from video. Computer Vision and Image Understanding, 91:214-245, 2003

[7] X. Liu and T. Chen. Video-based face recognition using adaptive hidden markov models. In Proc. Int. Conf. on Computer Vision and Pattern Recognition, 2003

[8] K.C. Lee, J. Ho, M.H. Yang, and D. Kriegman. Video-based face recognition using probabilistic appearance manifolds. In Proc. Int. Conf. on Computer Vision and Pattern Recognition, 2003.

[9] A. Hadid and M. Pietikäinen. An experimental investigation about the integration of facial dynamics in video-based face recognition. Electronic Letters on Computer Vision and Image Analysis, 5(1):1-13, 2005.

[10] A.J. OToole, D.A. Roark, and H. Abdi. Recognizing moving faces: A psychological and neural synthesis. Trends in Cognitive Science, 6:261266, 2002.

[11] C. Darwin. The expression of the emotions in man and animals. London, U.K.: John Murray, 1965. (Original work published 1872)

[12] C. Goren, M. Sarty, and P. Wu. Visual following and pattern discrimination of face-like stimuli by newborn infants. Pediatrics, vol. 56, 544-549, 1975.

[13] G. E. Walton, and T. G. R. Bower. Newborns form prototypes in less than 1 minute. Psychological Science, vol. 4, 203-205, 1993.

[14] J. Fagan. Infants recognition memory for face. Journal of Experimental Child Psychology, vol. 14, 453-476, 1972.

[15] M. de Haan, and C. A. Nelson. Recognition of the mothers face by 6-month-old infants: A neurobehavioral study. Child Development, vol. 68, 187-210, 1997.

[16] C. M. Leonard, E. T. Rolls, F. A. W. Wilson, and G. C. Baylis. Neurons in the amygdala of the monkey with responses selective for faces. Behavioral Brain Research, vol. 15, 159-176, 1985.

[17] Gauthier, M. J. Tarr, A. W. Anderson, P. Skudlarski, and J. C. Gore. Activation of the middle fusiform face area increases with expertise in recognizing novel objects. Nature Neuroscience, vol. 2, 568573, 1999.

[18] N. Kanwisher, J. McDermott, and M. M. Chun. The fusiform face area: A module in human extrastriate cortex specialized for face perception. Journal of Neuroscience, vol. 17, 43024311, 1997.

[19] G. McCarthy, A. Puce, J. C. Gore, and T. Allison. Facespecific processing in the human fusiform gyrus. Journal of Cognitive Neuroscience, vol. 8, 605610, 1997

[20] R. T. Schultz, I. Gauthier, A. Klin, R. K. Fulbright, A. W. Anderson, F. R. Volkmar, P. Skudlarski, C. Lacadie, D. J. Cohen, and J. C. Gore. Abnormal ventral temporal cortical activity during face discrimination among individuals with autism and Asperger syndrome. Archives of General Psychiatry, vol. 57, 331-340, 2000.

[21] A. R. Damasio, J. Damasio, and G. W. Van Hoesen. Prosopagnosia: Anatomic basis and behavioral mechanisms. Neurology, vol. 32, 331$341,1982$.

[22] C. A. Nelson. The development and neural bases of face recognition. Infant and Child Development, vol. 10, 3-18, 2001.

[23] J. P. Aggleton, M. J. Burton, and R. E. Passingham. Cortical and subcortical afferents to the amygdala of the rhesus monkey (Macaca mulatta). Brain Research, vol. 190, 347-368, 1980.
[24] J.V. Haxby, E.A. Hoffman, and M.I. Gobbini. The distributed human neural system for face perception. Trends in Cognitive Sciences, vol. 4, No. 6, 223-233, June 2000.

[25] Vaina, L.M., Solomon, J., Chowdhury, S., Sinha, P., Belliveau, J.W. Functional Neuroanatomy of Biological Motion Perception in Humans. In: Proc. of the National Academy of Sciences of the United States of America, Vol. 98, No. 20 (Sep. 25, 2001) , pp. 11656-11661

[26] M. Tistarelli, and E. Grosso. Active vision-based face authentication. Image and Vision Computing: Special issue on Facial Image Analysis, M. Tistarelli ed., vol. 18, no. 4, 299-314, 2000.

[27] M. Bicego, E. Grosso, and M. Tistarelli, "On finding differences between faces," in Audio- and Video-based Biometric Person Authentication, T. Kanade, A. Jain, and N.K. Ratha, Eds., vol. LNCS 3546, pp. 329-338. Springer, 2005

[28] L. Wiskott, J.M. Fellous, N. Kruger, and C. von der Malsburg. Face recognition and gender determination. In Proceedings Int.l Workshop on Automatic Face and Gesture Recognition, pp. 92-97, Zurich, Switzerland, 1995.

[29] H. Wechsler, P. Phillips, V. Bruce, F. Soulie, and T. Huang (Eds.). Face Recognition. From Theory to Applications, NATO ASI Series F, Vol. 163, Springer-Verlag, Berlin Heidelberg.

[30] G. Cottrell, and J. Metcalfe. Face, gender and emotion recognition using holons. In D. Touretzky, editor, Advances in Neural Information Processing Systems, Vol. 3, 564-571, San Mateo, CA, 1991. Morgan Kaufmann.

[31] B. Braathen and M. S. Bartlett and G. Littlewort and J. R. Movellan. First Steps Towards Automatic Recognition of Spontaneous Facial Action Units ACM Workshop on Perceptive User Interfaces, Orlando (FL), Nov. 15-16 2001.

[32] R.W. Picard. Toward computers that recognize and respond to user emotion. IBM System,(39), 3/4, 2000.

[33] R.W. Picard. Building HAL: Computers that sense, recognize, and respond to human emotion. MIT Media-Lab TR-532. Also in Society of Photo-Optical Instrumentation Engineers. Human Vision and Electronic Imaging VI, part of SPIE9s Photonics West, 2001.

[34] M. Bicego, E. Grosso, and M. Tistarelli. Person authentication from video of faces: a behavioral and physiological approach using Pseudo Hierarchical Hidden Markov Models. In Proc. of Intern.l Conference on Biometric Authentication 2006, 113-120, Hong Kong, China, 2006. Springer Verlag, LNCS 3832.

[35] L. Rabiner. A tutorial on Hidden Markov Models and selected applications in speech recognition. Proc. of IEEE, 77(2):257-286, 1989.

[36] V.V. Kohir and U.B. Desai. Face recognition using DCT-HMM approach. In Proc. Workshop on Advances in Facial Image Analysis and Recogniti Technology (AFIART), Freiburg, Germany, 1998.

[37] F. Samaria. Face recognition using Hidden Markov Models. PhD thesis, Engineering Department, Cambridge University, October 1994

[38] A.V. Nefian and M.H. Hayes. Hidden Markov models for face recognition. In Proc. Int. Conf. on Acoustics, Speech and Signal Processing (ICASSP), pages 2721-2724, Seattle, 1998.

[39] M. Bicego, U. Castellani, and V. Murino. Using Hidden Markov Models and wavelets for face recognition. In IEEE. Proc. of Int. Conf on Image Analysis and Processing, pages 52-56, 2003.

[40] M. Bicego, E. Grosso, and M. Tistarelli. Probabilistic face authentication using hidden markov models. In Proc. of SPIE Int. Workshop on Biometric Technology for Human Identification, 2005.

[41] S. Fine, Y. Singer, and N. Tishby. The hierarchical hidden markov model: Analysis and applications. Machine Learning, 32:41-62, 1998.

[42] P. Smyth. Clustering sequences with hidden Markov models. In M. Mozer, M. Jordan, and T. Petsche, editors, Advances in Neural Information Processing Systems, volume 9, page 648. MIT Press, 1997.

[43] A. Panuccio, M. Bicego, and V. Murino. A Hidden Markov modelbased approach to sequential data clustering. In Structural, Syntactic and Statistical Pattern Recognition, volume LNCS 2396, pages 734742. Springer, 2002.

[44] G. Schwarz. Estimating the dimension of a model. The Annals of Statistics, 6(2):461-464, 1978.

[45] L. Rabiner, C. Lee, B. Juang, and J. Wilpon. HMM clustering for connected word recognition. In Proc. Int. Conf. on Acoustics, Speech and Signal Processing (ICASSP), pages 405-408, 1989.

[46] C. Li. A Bayesian Approach to Temporal Data Clustering using Hidden Markov Model Methodology. PhD thesis, Vanderbilt University, 2000.

[47] A.K. Jain and R. Dubes. Algorithms for clustering data. Prentice Hall, 1988. 